\title{
ENANTIOSELECTIVE BIOTRANSFORMATION OF PROPRANOLOL TO THE ACTIVE METABOLITE 4-HYDROXYPROPRANOLOL BY ENDOPHYTIC FUNGI
}

\author{
Keyller Bastos Borges e Pierina Sueli Bonato* \\ Café, s/n, 14040-903 Ribeirão Preto - SP, Brasil \\ Mônica Tallarico Pupo \\ Av. do Café, s/n, 14040-903 Ribeirão Preto - SP, Brasil \\ Recebido em 22/11/10; aceito em 18/3/11; publicado na web em 10/6/11
}

Departamento de Física e Química, Faculdade de Ciências Farmacêuticas de Ribeirão Preto, Universidade de São Paulo, Av. do

Departamento de Ciências Farmacêuticas, Faculdade de Ciências Farmacêuticas de Ribeirão Preto, Universidade de São Paulo,

\begin{abstract}
The enantioselective biotransformation of propranolol (Prop) by the endophytic fungi Phomopsis sp., Glomerella cingulata, Penicillium crustosum, Chaetomium globosum and Aspergillus fumigatus was investigated by studying the kinetics of the aromatic hydroxylation reaction with the formation of 4-hydroxypropranolol (4-OH-Prop). Both Prop enantiomers were consumed by the fungi in the biotransformation process, but the 4-hydroxylation reaction yielded preferentially (-)-(S)-4-OH-Prop. The quantity of metabolites biosynthesized varied slightly among the evaluated endophytic fungi. These results show that all investigated endophytic fungi could be used as biosynthetic tools in biotransformation processes to obtain the enantiomers of 4-OH-Prop.
\end{abstract}

Keywords: enantioselective biotransformation; propranolol; endophytic fungi.

\section{INTRODUCTION}

In humans, cytochrome P450 is a superfamily of heme-containing enzymes that perform a great variety of reactions on a wide range of substrates, including pharmaceuticals. ${ }^{1}$ Some metabolites produced in the biotransformation reactions are biologically active themselves, and the study of their effects is crucial in evaluating drug's efficacy, toxicity, and pharmacokinetics. ${ }^{2}$ These studies, however, require large quantities of the pure metabolites, and in some cases, they may be difficult to synthesize. An alternative to chemical synthesis is to use biomimetic systems that reproduce the action of the P450s to generate the metabolites of the drugs or the drug candidates. Isolated enzymes or microorganisms (fungi, bacteria or yeasts) can be used in biotransformation processes to generate specific metabolites. ${ }^{3}$

Among the sources for microbial biotransformations, fungi seem to be promising due to the possibility of obtaining large quantities of the major and the minor metabolites. In addition, the use of these microorganisms has the advantages of biotransformation with lower cost, greater efficiency and in less time when compared to other biotransformation processes, such as with the use of experimental animals, mammalian enzyme systems or cell cultures. ${ }^{4}$ In addition, fungi can perform regio- and enantio-selective reactions, ${ }^{5}$ allowing to obtain a particular specie without the need of a laborious purification step.

Endophytic fungi is a term applied to those fungi that live within plant tissues, for all or part of their life cycle, and cause no apparent infections. ${ }^{6}$ Endophyte-host relationships are remarkable for the expression of a balance of antagonisms between endophytic virulence and plant defensive response. ${ }^{7}$ In recent years, the number of works that use endophytic fungi in biotransformation processes has also increased. ${ }^{8}$

Non selective $\beta$-adrenergic blockers are therapeutic agents that inhibit the effect of catecholamines on $\beta_{1}$ - and $\beta_{2}$-adrenergic recep-

*e-mail: psbonato@fcfrp.usp.br tors. One example of a common $\beta$-adrenergic blocker is propranolol (Prop), which has been prescribed to treat hypertension, tachyarrhythmia, angina pectoris, myocardial infarction and others. ${ }^{9}$ Prop is an aryl-substituted alkylamine, containing a naphthalene moiety which comprises its fluorophore and an alkyl side chain which has one chiral center. The $\beta$-blocking activity of $(-)-(S)$-Prop is about 100 times higher than the activity observed for $(+)-(R)$-Prop. ${ }^{10}$ The main metabolite 4-hydroxypropranolol (4-OH-Prop) is also pharmacologically active (see Figure 1 compound structures). ${ }^{11}$ Since (-)-(S)-OH-Prop is also pharmacologically active, it would be interesting to obtain this active metabolite by a biotransformation process.

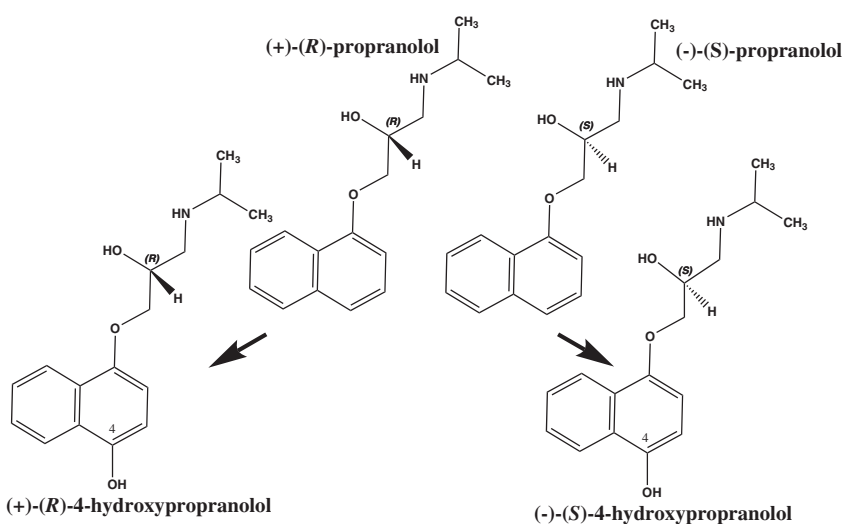

Figure 1. Chemical structures of propranolol and 4-hydroxypropranolol enantiomers. *Chiral center

The microbial biotransformation of Prop was previously studied by Foster et al.. ${ }^{12}$ The fungus Cunninghamella bainieri biotransformed Prop to 4-OH-Prop and the metabolites desisopropylpropranolol, propranolol glycol, 1-naphthoxylactic acid and 1-naphthoxyacetic acid were also found in trace amounts. C. echinulata was also used to 
biotransform Prop, ${ }^{13}$ but the observed profile was different because the major metabolite formed was identified as 8-hydroxypropranolol. In addition, the authors reported an enantioselective biotransformation by the fungus, which initially metabolized $(-)-(S)$-Prop in a faster way and to a greater extention to 8-hydroxypropranolol than to $(+)-(R)$-Prop.

Based on these concerns, Prop was selected as a model analyte to study the aromatic hydroxylation reaction by some endophytic fungi. The reaction was followed by using an enantioselective method, therefore, the enantioselectivity of the process of Prop biotransformation to its active metabolite 4-hydroxypropranolol could be evaluated.

\section{EXPERIMENTAL}

\section{Microorganisms}

The endophytic fungi have been isolated and identified by their rDNA sequences as previously described..$^{14}$ The selected strains were Phomopsis sp. (TD2) isolated from Tithonia diversifolia; Glomerella cingulata (VA1) isolated from Viguiera arenaria; Penicillium crustosum (VR4), Chaetomium globosum (VR10) and Aspergillus fumigatus (VR12) isolated from Viguiera robusta. The fungi have been deposited in the Academic Laboratory of Chemistry of Microorganisms of Faculty of Pharmaceutical Sciences of Ribeirão Preto (University de São Paulo, Ribeirão Preto, Brazil) and have been maintained as agar plugs in sterile water, in silica gel (6 to 12 mesh, grade 40 , desiccant activated) at $10{ }^{\circ} \mathrm{C}$, or on potato dextrose agar (PDA) slants and stored at $4{ }^{\circ} \mathrm{C}$.

\section{Chemicals}

Rac-propranolol (99\%) was obtained from Sigma Aldrich (St. Louis, USA) and rac-4-hydroxypropranolol (98\%) was obtained from Toronto Research Chemicals (North York, Canada). Carboxymethyl$\beta$-cyclodextrin (CM- $\beta-C D)$ was obtained from Fluka (Buchs, Switzerland). Acetaminophen (Acet, 98\%) obtained from Sigma Aldrich (St. Louis, USA) was used as internal standard on the analytical procedure. Triethylamine (TEA) was purchased from J.T. Baker (Phillipsburg, USA) and phosphoric acid $\left(\mathrm{H}_{3} \mathrm{PO}_{4}, 85 \%\right.$ in water) was obtained from Labsynth (Diadema, Brazil). Sodium hydroxide was purchased from Nuclear (Diadema, Brazil). Diethyl ether was purchased from Mallinckrodt Baker (Paris, USA) and ethyl acetate from Tedia (Fairfield, USA). All other chemicals were of analyticalgrade in the highest purity available. Water was distilled and purified using a Millipore Milli-Q Plus system (Bedford, USA).

\section{Biotransformation conditions}

\section{Step I}

Three discs of $0.5 \mathrm{~cm}$ of diameter containing the fungal mycelia were aseptically transferred to $9.0 \mathrm{~cm}$ diameter Petri dishes containing PDA and allowed to grow for 7 days at $25^{\circ} \mathrm{C}$.

Step II

Three uniform discs of $0.5 \mathrm{~cm}$ diameter of each endophytic fungus mycelia studied were cut with a transfer tube (Fischer Scientific, Pittsburgh, USA) and then inoculated in $50 \mathrm{~mL}$ Falcon tubes containing $10 \mathrm{~mL}$ of pre-fermentative medium (10.0 g malt extract, 10.0 $\mathrm{g}$ dextrose, $5.0 \mathrm{~g}$ triptone and $3.0 \mathrm{~g}$ yeast extract and distilled water to $1 \mathrm{~L}$ and $\mathrm{pH}$ adjusted to $6.2 \pm 0.2$ with a solution of $0.5 \mathrm{~mol} \mathrm{~L}^{-1}$ $\mathrm{HCl})$ that was used for the appropriate growth of the microorganism.

Step III

The mycelium obtained after three days was completely trans- ferred to $250 \mathrm{~mL}$ Erlenmeyer flask containing $100 \mathrm{~mL}$ of modified Czapek medium (25.0 g sucrose, $2.0 \mathrm{~g} \mathrm{NaNO}_{3}, 1.0 \mathrm{~g} \mathrm{KH}_{2} \mathrm{PO}_{4}, 0.5$ $\mathrm{g} \mathrm{MgSO}_{4} \cdot 7 \mathrm{H}_{2} \mathrm{O}, 0.5 \mathrm{~g} \mathrm{KCl}, 0.01 \mathrm{~g} \mathrm{FeSO}_{4} \cdot 7 \mathrm{H}_{2} \mathrm{O}$, and distilled water to $1.0 \mathrm{~L}, \mathrm{pH}$ adjusted to 5.0 with a solution of $1.0 \mathrm{~mol} \mathrm{~L}^{-1} \mathrm{HCl}$ ). At this point, rac-Prop ( $2 \mathrm{mg}$ ) was dissolved in $100 \mu \mathrm{L}$ of $N, N$ dimethylformamide and added to modified Czapek medium. This solution was filter-sterilized with a disposable $0.22 \mu \mathrm{m}$ membrane filter (Millipore, São Paulo, Brazil) and the exact concentration of Prop was determined using the CE method. The quantification of Prop was necessary due to the drug loss by adsorption onto the filter matrix. The cultures were incubated for $144 \mathrm{~h}$ at $30^{\circ} \mathrm{C}$, in the darkness, with shaking at $120 \mathrm{rpm}$.

\section{Enantioselective capillary electrophoretic method}

The analytical method used for the determination of Prop and 4-OH-Prop enantiomers by capillary electrophoresis was previously described by our group. ${ }^{8}$ In summary, the analytes were extracted from the culture medium using a mixture of diethyl ether: ethyl acetate $(1: 1, v / v)$ and the analyses were performed on an Agilent Technologies CE system (Waldbronn, Germany) model G1600A, consisting of an analyzer, an automatic sampler and a diode array detector. A fused-silica uncoated capillary obtained from MicroSolv Technology Corporation (Eatontown, USA) of $50 \mu \mathrm{m}$ id, $50.0 \mathrm{~cm}$ in total length, and $41.5 \mathrm{~cm}$ in effective length was used. The electrophoretic conditions were: $4 \%(w / v) \mathrm{CM}-\beta-\mathrm{CD}$ in $25 \mathrm{mmol} \mathrm{L} \mathrm{L}^{-1} \mathrm{TEA} / \mathrm{H}_{3} \mathrm{PO}_{4}$ buffer at $\mathrm{pH} 9$ as running electrolyte and $17 \mathrm{kV}$ of voltage. UV detection was carried out at $208 \mathrm{~nm}$. Sample injections were performed hydrodynamically at a pressure of 50 mbar for 20 s and the capillary temperature was set at $25^{\circ} \mathrm{C}$.

Calibration curves were obtained by spiking aliquots of $1 \mathrm{~mL}$ liquid Czapek medium with standard solutions of rac-Prop and rac-4-OH-Prop to obtain enantiomer concentrations in the range of $0.10-10.0$ and $0.25-10.0 \mu \mathrm{g} \mathrm{mL}{ }^{-1}$, respectively. The internal standard (acetaminophen) was used in the concentration of $100 \mu \mathrm{g} \mathrm{mL}^{-1}$.

\section{RESULTS AND DISCUSSION}

The use of microbial models to study the biotransformation of drugs have several advantages over studies with animals and humans, due to lower cost, ease of setup and manipulation, and higher yield and diversity of metabolite production. Microbial metabolite production is especially important for metabolites that are hardly synthesized by organic chemical methods. An important goal in microbial model studies is to develop a list of microorganisms that can mimic the metabolic profiles of drugs in mammals.

In our previous work reporting the development and validation of the enantioselective method for the analysis of Prop and 4-OHProp in culture medium, we also showed the results obtained for the biotransformation reaction after 3 days of incubation. ${ }^{8}$ In the present paper, we are presenting the kinetic study of Prop biotransformation by some endophytic fungi. This investigation permitted to monitor the obtained metabolite concentrations versus time and the formation and consumption of formed metabolites. In addition, we could observe the formation of other metabolic species that were not monitored.

All endophytic fungi studied were able to enantioselectively biotransform Prop to 4-OH-Prop, the active metabolite. The percentage of metabolites produced was calculated considering the Prop amount (2 $\mathrm{mg}$ ) that was added in $100 \mathrm{~mL}$ of culture medium $\left(20 \mu \mathrm{g} \mathrm{mL}^{-1}\right)$, which resulted in concentrations of $10 \mu \mathrm{g} \mathrm{mL} \mathrm{L}^{-1}$ of each enantiomer, $(+)-(R)$ Prop and (-)-(S)-Prop. Generally, the concentrations of 4-OH-Prop enantiomers increased during the first 3 days and then they began to decrease slowly for the next 4 days, since these metabolites had also 
started to be consumed and transformed to other products, as seen in the biotransformation process employing Phomopsis sp. (TD2). Figure 2 shows the typical electropherograms obtained from samples of the investigated biotransformation process. Noninoculated controls and cultures with Prop absence did not present peaks corresponding neither to any of the enantiomers nor to other unknown compounds.

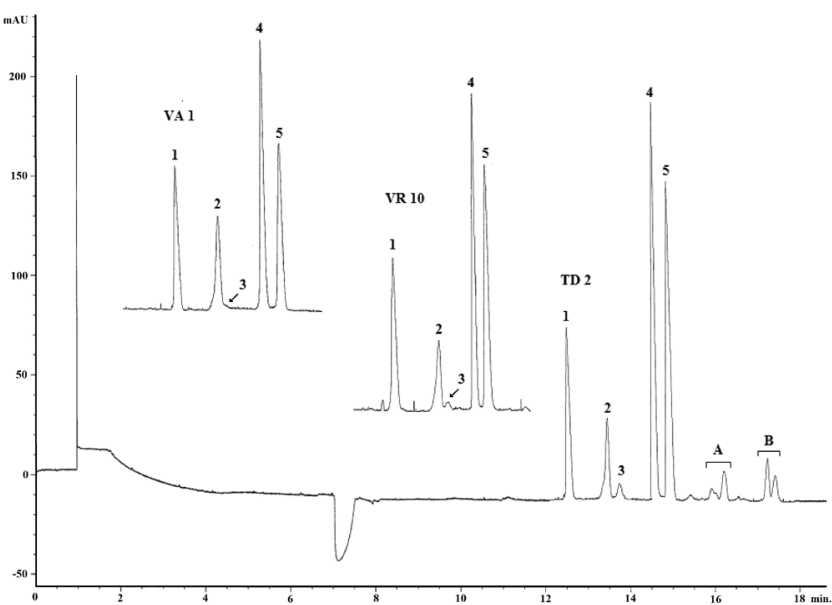

Figure 2. Electropherograms of some endophytic fungi studied: Glomerella cingulata (VA1) after 144 h of incubation, Chaetomium globosum (VR10) after $144 \mathrm{~h}$ of incubation and Phomopsis sp. after $72 \mathrm{~h}$ of incubation (TD2), (1) Acet, (2) (-)-(S)-4-OH-Prop, (3) (+)-(R)-4-OH-Prop, (4) (-)-(S)-Prop and $(5)(+)-(R)-P r o p$. Electrophoretic conditions: buffer concentration of $25 \mathrm{mmol}$ $L^{-1}$, buffer $\mathrm{pH} 9,4 \%(w / v)$ of $C M-\beta-C D$ and voltage of $17 \mathrm{kV}$

Phomopsis sp. (TD2) was able to biotransform (-)-(S)- and $(+)-(R)$-Prop; $45 \%$ of $(-)-(S)$ - Prop added to the reaction medium

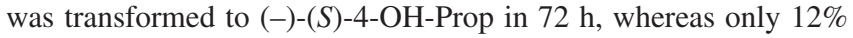
of $(+)-(R)$-Prop was converted to $(+)-(R)-4-\mathrm{OH}-$ Prop in this period of incubation (Figure 3a). The enantioselectivity in the production of the metabolite was lost in longer analysis time. In addition, other unidentified metabolites were also observed for this fungus. So, Phomopsis sp. (TD2) is a fungus that deserves more studies using higher amounts of substrate (Prop) for the isolation and characterization of the unknown metabolites (A and B, Figure 2).

Glomerella cingulata (VA1) biotransformed $29.1 \%$ and $47.7 \%$ of (-)-(S)-Prop to (-)-(S)-4-OH-Prop within 24 and $72 \mathrm{~h}$ of incubation, respectively (Figure $3 \mathrm{~b})$. The $(+)-(R)-4-\mathrm{OH}-\mathrm{Prop}$ metabolite had not been formed before $72 \mathrm{~h}$ of incubation, showing that this fungus is very interesting for the production of the active enantiomer of the metabolite. The highest concentration $\left(0.83 \mu \mathrm{g} \mathrm{mL}^{-1}\right)$ of $(+)-(R)-4-\mathrm{OH}-$ Prop metabolite was observed in $120 \mathrm{~h}$ of incubation (Figure 3b).

The other three fungi, Penicillium crustosum (VR4), Chaetomium globosum (VR10) and Aspergillus fumigatus (VR12) showed similar metabolic profile (Figure $3 \mathrm{c}-\mathrm{d}$ ), except for longer incubation periods. In this case, both Chaetomium globosum (VR10) and Aspergillus fumigatus (VR12) showed higher enantioselectivitiy in the production of the metabolite (-)-(S) after $144 \mathrm{~h}$ of incubation. In $48 \mathrm{~h}$ of incubation, Chaetomium globosum (VR10) presented the formation of $24.1 \%$ of (-)-(S)-4-OH-Prop and it did not form (+)-(R)-4-OHProp (Figure 3d). With longer periods of incubation with Aspergillus fumigatus (VR12), it was evidenced higher enantioselectivity for the formation of (-)-(S)-4-OH-Prop. In general, 38.3 to $45.1 \%$ of (-)-(S)Prop were transformed to (-)-(S)-4-OH-Prop, and 23.1 to $29.8 \%$ of $(+)-(R)$-Prop were transformed to $(+)-(R)-4-\mathrm{OH}-$ Prop.

In humans, CYP2D6 and CYP1A2 are the major P450s responsible for the biotransformation of Prop ${ }^{15}$ to the different products.
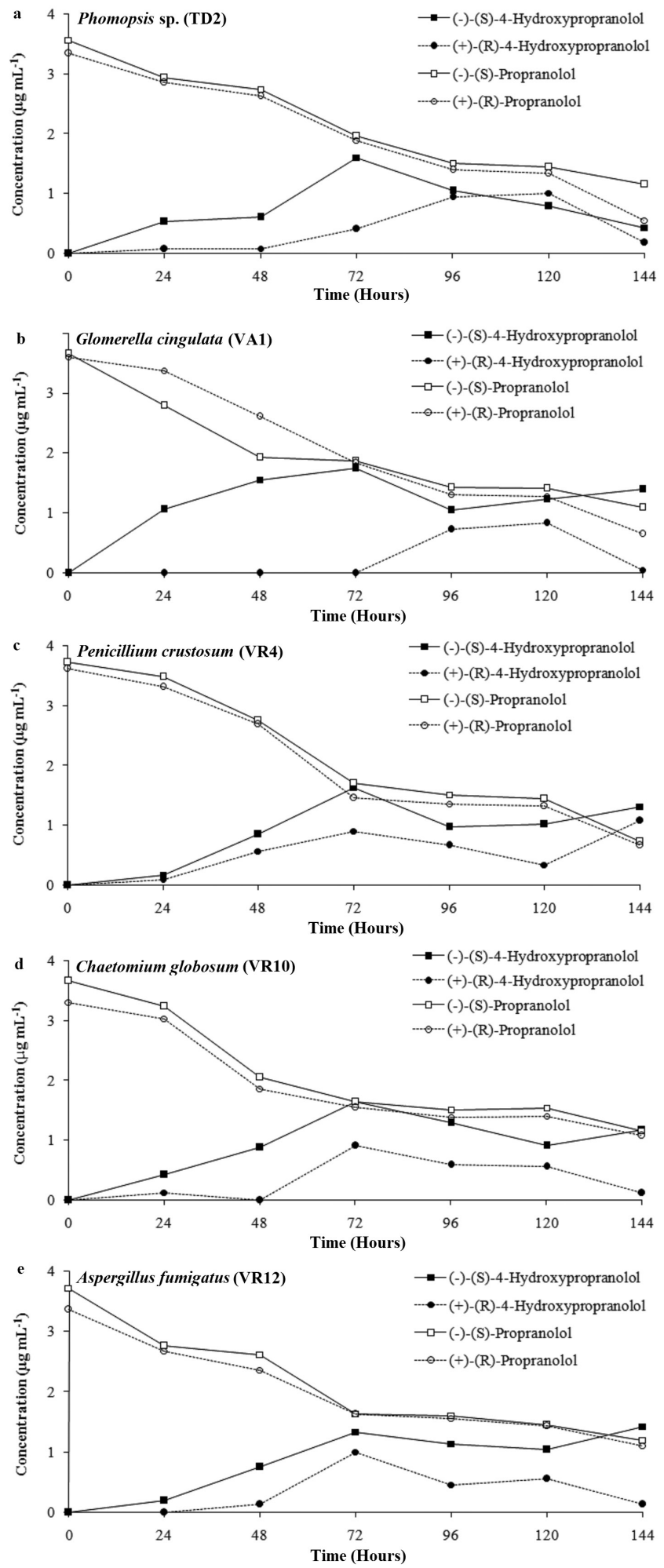

Figure 3. Concentration-time profile of PROP and 4-OH-PROP enantiomers in modified Czapek medium with (a) Phomopsis sp. (TD2); (b) Glomerella cingulata (VA1); (c) Penicillium crustosum (VR4), (d) Chaetomium globosum (VR10), and (e) Aspergillus fumigatus (VR12)

The ring-hydroxylated products (4-OH-Prop and 5-OH-Prop) are of particular importance due to their pharmacological activities. ${ }^{16}$ The study of these microorganisms in semi-preparative or preparative scale can be useful to isolate the metabolites from culture medium in bigger amounts. 
Finally, as a suitable alternative to Cunninghamella species, the endophytic fungi could be used as complementary models for predicting in vivo metabolism and to produce metabolites of drugs, that could be used for bionalytical method development and other applications.

\section{CONCLUSIONS}

The results obtained here show that the used endophytic fungi biotransformed Prop trough 4-hydroxylation, as it happens in mammals. In addition, the 4-hydroxilation occurred preferentially with the formation of the $S$-enantiomer, the more active metabolite. Endophytic fungi have proven to be a valuable tool to predict in vivo metabolism and to produce quantities of metabolites to be used as reference standard for bionalytical method development and for other applications.

\section{ACKNOWLEDGEMENTS}

The authors are grateful to Fundação de Amparo à Pesquisa do Estado de São Paulo (FAPESP), Conselho Nacional de Desenvolvimento Científico e Tecnológico $(\mathrm{CNPq})$ and to Coordenação de Aperfeiçoamento de Pessoal de Nível Superior (CAPES) for financial support and for granting research fellowships.

\section{REFERENCES}

1. Lewis, D. F. V.; Guide to Cytochromes P450: structure and function, Taylor \& Francis: New York, 2001; Guengerich, F. P.; Mol. Interv. 2003 3, 194.

2. Fura, A.; Drug Discov. Today 2006, 11, 133.

3. Pupo, M. T.; Borges, K. B.; Borges, W. S.; Bonato, P. S. In Microbial Biotechnology; Saikai, R.; Bezbaruah, R. L.; Bora, T. Ch., eds.; New India Publishing Agency: New Delli, 2008, chap. 3.

4. Borges, W. S.; Borges, K. B.; Bonato, P. S.; Said, S.; Pupo, M. T.; Cur. Org. Chem. 2009, 13, 1137.

5. Borges, K. B.; Borges, W. S.; Durán-Patrón, R.; Pupo, M. T.; Bonato, P. S.; Collado, I. G.; Tetrahedron: Asymmetry 2009, 20, 385.

6. Wilson, D.; Oikos 1993, 68, 379; Wennström, A.; Oikos 1994, 71, 535.

7. Schulz, B.; Boyle, C.; Mycol. Res. 2005, 109, 661; Tan, R. X.; Zou, W. X.; Nat. Prod. Rep. 2001, 18, 448.

8. Werner, C.; Petrini, O.; Hesse, M.; FEMS Microbiol. Lett. 1997, 155, 147; Yue, Q.; Bacon, C. W.; Richardson, M. D.; Phytochemistry 1998,
48, 451; Tanaka, M.; Yoshimura, M.; Suto, M.; Yokota, A.; Asano, K.; Sukara, E.; Tomita, F.; J. Biosci. Bioeng. 2002, 93, 531; Zikmundová, M.; Drandarov, K.; Bigler, L.; Hesse, M.; Werner, C.; Appl. Environ. Microbiol. 2002, 68, 4863; Zikmundová, M.; Drandarov, K.; Hesse, M.; Werner, C. Z.; Z. Naturforsch, C: J. Biosci. 2002, 57, 660; Glenn, A. E.; Meredith, F. I.; Morrison, III, W. H.; Bacon, C. W.; Appl. Environ. Microbiol. 2003, 69, 3165; Shibuya, H.; Kitamura, C.; Maehara, S.; Nagahata, M.; Winarno, H., Simanjuntak, P.; Kim, H.-S.; Wataya, Y.; Ohashi, K.; Chem. Pharm. Bull. 2003, 51, 71; Agusta, A.; Maehara, S.; Ohashi, K.; Simanjutak, P.; Shibuya, H.; Chem. Pharm. Bull. 2005, 53, 1565; Shibuya, H.; Agusta, A.; Ohashi, K.; Maehara, S.; Simanjuntak, P.; Chem. Pharm. Bull. 2005, 53, 866; Borges, K. B.; Borges, W. S.; Pupo, M. T.; Bonato, P. S.; Appl. Microbiol. Biotechnol. 2007, 77, 669; Borges, K. B.; Borges, W. S.; Pupo, M. T.; Bonato, P. S.; J. Pharm. Biomed. Anal. 2008, 46, 945; Verza, M.; Arakawa, N. S.; Lopes, N. P.; Kato, M. J.; Pupo, M. T.; Said, S.; Carvalho, I.; J. Braz. Chem. Soc. 2009, 20, 195; Borges, K. B.; Pupo, M. T.; Bonato, P. S.; Electrophoresis 2009, 30, 3910; Borges, K. B.; Okano, L. T.; Pupo, M. T.; Bonato, P. S.; Chromatographia 2009, 70, 1335; Barth, T.; Pupo, M. T.; Borges, K. B.; Okano, L. T.; Bonato, P. S.; Electrophoresis 2010, 31, 1521; Borges, K. B.; de Oliveira, A. R. M.; Barth, T.; Jabor, V. A. P.; Pupo, M. T.; Bonato, P. S.; Anal. Bioanal. Chem. 2011, 399, 915.

9. Rossi, S.; Australian Medicines Handbook, Australian Medicines Handbook: Adelaide, 2006.

10. Barrett, A. M.; Cullum, V. A.; Br. J. Pharmacol. 1968, 34, 43; Walle, T.; Drug Metab. Dispos. 1985, 13, 279.

11. Riopel, D. A.; Walle, T.; Clin. Pharmacol. Ther. 1980, 28, 743.

12. Foster, B. C.; Buttar, H. S.; Qureshi, S. A.; McGilveray, J.; Xenobiotica 1989, 19, 539

13. Foster, B. C.; Litster, D. L.; Wilson, D. L.; Ormsby, E.; Dawson, B. A.; Xenobiotica 1992, 22, 1221.

14. Guimarães, D. O.; Borges, W. S.; Kawano, C. Y.; Ribeiro, P. H.; Goldman, G. H.; Nomizo, A.; Thiemann, O. H.; Oliva, G.; Lopes, N. P.; Pupo, M. T.; FEMS Immunol. Med. Microbiol. 2008, 52, 134; Momesso, L. S.; Kawano, C. Y.; Ribeiro, P. H.; Nomizo, A.; Goldman, G. H.; Pupo, M. T.; Quim. Nova 2008, 31, 1680.

15. Masubuchi, Y.; Hosokawa, S.; Horie, T.; Suzuki, T.; Ohmori, S.; Kitada, M.; Narimatsu, S.; Drug Metab. Dispos. 1994, 22, 909.

16. Fitzgerald, J. D.; O’Donnell, S. R.; Br. J. Pharmacol. 1971, 43, 222; Oatis, J. E. Jr.; Russell, M. P.; Knapp, D. R.; Walle, T. J.; Med. Chem. 1981, 24, 309 . 